\title{
CLINICAL RESEARCH ARTICLE Blood pressure extremes and severe IVH in preterm infants
}

\author{
Zachary A. Vesoulis ${ }^{1}$, Abigail A. Flower ${ }^{2}$, Santina Zanelli ${ }^{3}$, Ami Rambhia ${ }^{1}$, Maryam Abubakar ${ }^{3}$, Halana V. Whitehead ${ }^{1}$, \\ Karen D. Fairchild ${ }^{3}$ and Amit M. Mathur ${ }^{1}$
}

BACKGROUND: The optimal upper and lower limits of blood pressure in preterm infants are not known. Exceeding these thresholds may contribute to intraventricular hemorrhage (IVH).

METHODS: Preterm infants born $\leq 30$ weeks GA were identified. Infants had continuous measurement of mean arterial blood pressure (MABP) for 7 days and cranial ultrasound imaging. IVH was classified as severe IVH (grade 3/4), no severe IVH (no IVH; grade 1/2), or no IVH. Mean \pm SEM MABP values from hours 1-168 were calculated and sorted into bins $2 \mathrm{~mm} H \mathrm{Hg}$ wide. The normalized proportion of each recording spent in each bin was then calculated. Candidate limits were identified by comparison of MABP distribution in those with severe IVH vs. those without severe IVH.

RESULTS: Eighty-five million measurements were made from 157 infants. Mean EGA was 25.2 weeks; mean BW was 749 g; 65/157 female; inotrope use in 59/157; grade 3/4 IVH in 29/157. Infants with severe IVH spent significantly more time with extreme MABP measurements $(<23 \mathrm{~mm} \mathrm{Hg}$ or $>46 \mathrm{~mm} \mathrm{Hg}$ ) compared to those without severe IVH $(12 \%$ vs. $8 \%$ of recording, $p=0.02)$.

CONCLUSIONS: Infants who developed severe IVH had substantially more unstable MABP and spent a significantly greater period of time with MABP outside of the optimal range.

Pediatric Research (2020) 87:69-73; https://doi.org/10.1038/s41390-019-0585-3

\section{INTRODUCTION}

Despite advances in neonatal care, intraventricular hemorrhage (IVH) remains a common complication of prematurity and the source of potentially severe neurodevelopmental consequences. ${ }^{1}$ Although rates of IVH have declined, owing largely to the use of antenatal steroids, ${ }^{2}$ it remains a persistent concern, affecting approximately $25 \%$ of all infants born before 30 weeks gestation. ${ }^{3}$ Despite efforts to reduce rates of IVH through "bundles" of clinical care practices, rates have remained essentially unchanged.

Our understanding of the mechanism(s) behind IVH has evolved over time, with a significant amount of recent research focused on the role of cerebrovascular autoregulation in the development of hemorrhage. ${ }^{4-7}$ In these studies, concurrent proxy measures of systemic blood flow (arterial blood pressure (BP)) and cerebral blood flow (derived by near-infrared spectroscopy (NIRS)) were used to quantify the function of the cerebrovascular autoregulatory system. Although important from a mechanistic standpoint, this approach remains abstracted from actionable clinical changes and requires continuous NIRS monitoring, often a challenge in the most preterm of infants with extremely fragile skin. A more clinically accessible target is the arterial BP, a value which is correlated with cerebral oxygenation. ${ }^{8}$ There has been extensive description of "normative" BP values in this population, yet these studies have yielded conflicting results, largely due to varying and inconsistent methodologic approaches. ${ }^{9-14}$ The optimal BP range in very low birth weight (BW) infants is unclear but might be a more easily accessed physiologic biomarker of brain injury risk.

We hypothesize that the risk of IVH is directly related to the length of time spent with BP exceeding upper and lower thresholds. The objective of this study was to empirically derive these thresholds, define the optimal arterial BP range, and to evaluate the relationship between the burden of BP extremes and severe IVH.

\section{METHODS}

Patient selection

Patients were selected from a prospectively recorded, multiinstitutional library of physiologic data collected from two level IV neonatal intensive care units (NICUs; St. Louis Children's Hospital, University of Virginia Children's Hospital) over a 5-year period (2012-2017). Both units provide care to a mix of in-born and outborn infants. Inclusion criteria were estimated gestational age (GA) $\leq 30$ weeks, cranial ultrasound imaging in the first week of life, and a minimum of 7 days of arterial BP data (umbilical or peripheral arterial line). Infants were excluded if they did not meet the above requirements or if they were transferred or expired prior to the end of the first week of life. The Institutional Review Board at each center reviewed and approved the study protocol.

\section{Sample characteristics}

Comprehensive demographic and outcome data were collected for all included infants, including GA at birth, BW, small for GA (defined as BW at <10th centile), sex, race, highest grade of $\mathrm{IVH}$, highest mode of ventilatory support, and mode of delivery. Exposure to inotropic medication (i.e., dopamine, epinephrine) and antenatal steroids was recorded. All infants had cranial ultrasound imaging performed at least once in the first week of life, and the highest noted grade of IVH was used for analysis.

\footnotetext{
${ }^{1}$ Division of Newborn Medicine, Department of Pediatrics, Washington University School of Medicine, St. Louis, MO, USA; ${ }^{2}$ Department of Systems and Information Engineering, Data Science Institute, University of Virginia, Charlottesville, VA, USA and ${ }^{3}$ Division of Neonatology, Department of Pediatrics, University of Virginia, Charlottesville, VA, USA Correspondence: Zachary A. Vesoulis (vesoulis_z@wustl.edu)
} 
BP data collection

At each center, physiologic data (including mean arterial BP (MABP)) from the patient monitor was prospectively archived in a database (BedMasterEx, ExcelMedical, Jupiter, FL) with a sampling rate of $0.5 \mathrm{~Hz}$. The file associated with each infant was then extracted from the database and converted to a MATLAB matrix for further processing.

\section{Pre-processing}

All data underwent a simple one-step error correction process, which removed out of range values (MABP $<15 \mathrm{~mm} \mathrm{Hg}$ or $>80$ $\mathrm{mm} \mathrm{Hg}$ ) as occurs during blood sampling or infusion. Next, the data were aligned by postnatal age (hours since birth) and the mean MABP for each hour was calculated. All measured mean MABP values were then sorted into 1 of the 15 bins, each 2-mm$\mathrm{Hg}$ wide, from $20-21 \mathrm{~mm} \mathrm{Hg}$ to $48-49 \mathrm{~mm} \mathrm{Hg}$. To account for variation in recording length, the proportion of time spent in each bin was normalized by recording length.

Empirically defining optimal BP

Current literature provides conflicting guidance as to the ideal MABP of a preterm infant and three divergent theories have emerged. In one proposal, a target MABP threshold of $30 \mathrm{~mm} \mathrm{Hg}$ emerged after a series of studies suggested a lower incidence of brain injury when MABP remains above this level. ${ }^{10,12,15} \mathrm{~A}$ second approach, based on normative data, calls for a target MABP threshold at or above the GA in weeks. ${ }^{9} A$ third approach, often called "permissive hypotension," is not focused on hard thresholds but rather is tolerant of low MABP with the hypothesis that the link between MABP and brain injury in tenuous at best. ${ }^{16}$

Although there is disagreement over the value of these bounds, there are unequivocal BP limits, beyond which there is pathologic cerebral blood flow. ${ }^{17-19}$ We hypothesized that infants with severe brain injury (IVH) will spend a greater proportion of the time with MABP values at the extremes of the distribution and that there is a direct correlation between the temporal burden of MABP extremes and the risk of severe IVH.

First, infants were divided into three groups by IVH status: severe IVH (grade 3 or 4 ), no severe IVH (no IVH or grades 1-2), or no detectable IVH. Next, the mean normalized proportion of the total recording length was averaged by group for each MABP bin. In order to identify candidate MABP thresholds, beyond which there is increased risk of severe IVH, we systematically examined differences in the distribution of BPs between the groups.

Statistical approach

Univariate analysis of clinical, demographic, and physiologic characteristics was conducted using Fisher's Exact test (categorical variables), Mann-Whitney test (two-group continuous variables), or Kruskal-Wallis test (three-group continuous variables).

To identify the optimal thresholds in an unbiased fashion, we serially examined the time spent in each MABP bin with a univariate binary logistic regression model in order to determine at which threshold there was the greatest discrimination between groups, defined by greatest area under the curve (AUC). As IVH itself may cause hemodynamic instability, this calculation was performed twice-first with a comparison of the severe IVH and not severe IVH groups followed by a comparison of severe IVH to no detectable IVH.

Although the initial thresholds were identified by comparing infants with and without severe IVH to maximize the sensitivity of the threshold, we hypothesized that there is in fact a dose-response relationship between the burden of MABP extremes and the severity of IVH. To this end, we developed a multivariate linear regression model to investigate the impact of MABP extreme burden on the severity of IVH (graded from 0 to 4) while controlling for other factors known to influence IVH (GA, BW, antenatal steroids, and inotrope exposure).
All statistical analysis was conducted in $R$ version 3.6.0 ( $R$ Foundation for Statistical Computing, Vienna, Austria).

\section{RESULTS}

Cohort characteristics

During the study period, 1392 infants were born at or before 30 completed weeks of gestation, 157 of which met all inclusion criteria. For the included infants, the mean EGA was $25.2 \pm$ 1.8 weeks; mean BW was $749 \pm 201 \mathrm{~g} ; 65 / 157$ female; inotrope use in $59 / 157$. IVH of any grade was present in $61 / 157$ (38\%) of the infants; grade $3 / 4$ was present in $29 / 157$ (18\%) of the infants and was diagnosed at a median age of $47.5 \mathrm{~h}$. Inotrope exposure differed between infants with and without severe IVH $(69 \%$ vs. $30 \%, p<0.01)$ as did documented antenatal steroid administration (62\% vs. $77 \%, p=0.02)$. A complete listing of infants' characteristics is shown in Table 1.

Data quality

A total of 85 million measurements from the 157 infants were included in the final analysis; a mean of $10 \%$ of the data were rejected for errors. The overall median recording length was $163 \mathrm{~h}$ and was not different between groups ( $153 \mathrm{~h}$ for no severe IVH, $143 \mathrm{~h}$ for severe IVH groups, $p=0.35$ ).

Table 1. Sample and recording characteristics

\begin{tabular}{|c|c|c|c|}
\hline & $\begin{array}{l}\text { No severe IVH } \\
(n=128)\end{array}$ & $\begin{array}{l}\text { Severe IVH } \\
(n=29)\end{array}$ & $p$ Value \\
\hline GA, mean (SD), weeks & $25.2(1.9)$ & $25.1(1.5)$ & 0.92 \\
\hline Birth weight, mean (SD), g & $741(203)$ & $786(195)$ & 0.21 \\
\hline Male sex, $n(\%)$ & $72(56)$ & $20(69)$ & 0.30 \\
\hline $\begin{array}{l}\text { Inotrope exposure in first } \\
7 \text { days, } n(\%)\end{array}$ & $39(30)$ & $20(69)$ & $<0.01^{*}$ \\
\hline Antenatal steroids, $n$ (\%) & $99(77)$ & $18(62)$ & $0.02^{*}$ \\
\hline Vaginal delivery, $n(\%)$ & $52(41)$ & $13(45)$ & 0.68 \\
\hline \multicolumn{4}{|l|}{ Race/ethnicity } \\
\hline Asian, $n(\%)$ & $1(1)$ & $0(0)$ & 0.29 \\
\hline Black, $n(\%)$ & $32(25)$ & $12(41)$ & \\
\hline Hispanic, $n$ (\%) & $7(5)$ & $1(4)$ & \\
\hline White, $n$ (\%) & $79(62)$ & $13(45)$ & \\
\hline Not listed, $n(\%)$ & $9(7)$ & $3(10)$ & \\
\hline \multicolumn{4}{|l|}{$\begin{array}{l}\text { Highest mode of } \\
\text { ventilatory support, } n(\%)\end{array}$} \\
\hline CPAP & $20(15)$ & $4(14)$ & 0.09 \\
\hline $\begin{array}{l}\text { Conventional } \\
\text { mechanical ventilation }\end{array}$ & $75(59)$ & $11(38)$ & \\
\hline $\begin{array}{l}\text { High-frequency } \\
\text { oscillatory ventilation }\end{array}$ & $33(26)$ & $14(48)$ & \\
\hline $\begin{array}{l}\text { Mean MABP, mean } \\
\text { (SD), } \mathrm{mm} \mathrm{Hg}\end{array}$ & $34.0(4.6)$ & $34.2(4.4)$ & 0.87 \\
\hline $\begin{array}{l}\text { Recording with MABP }< \\
G A \text {, mean }(S D), \%\end{array}$ & $14(13)$ & $26(29)$ & 0.48 \\
\hline $\begin{array}{l}\text { Recording with MABP }<30 \\
\mathrm{~mm} \mathrm{Hg} \text {, mean (SD), } \%\end{array}$ & $58(30)$ & $67(21)$ & 0.29 \\
\hline $\begin{array}{l}\text { Raw recording length, } \\
\text { median (IQR), } h\end{array}$ & $153(151-166)$ & $143(146-167)$ & 0.35 \\
\hline $\begin{array}{l}\text { Recording rejected for } \\
\text { errors, mean (SD), \% }\end{array}$ & $9(25)$ & $15(24)$ & 0.35 \\
\hline
\end{tabular}




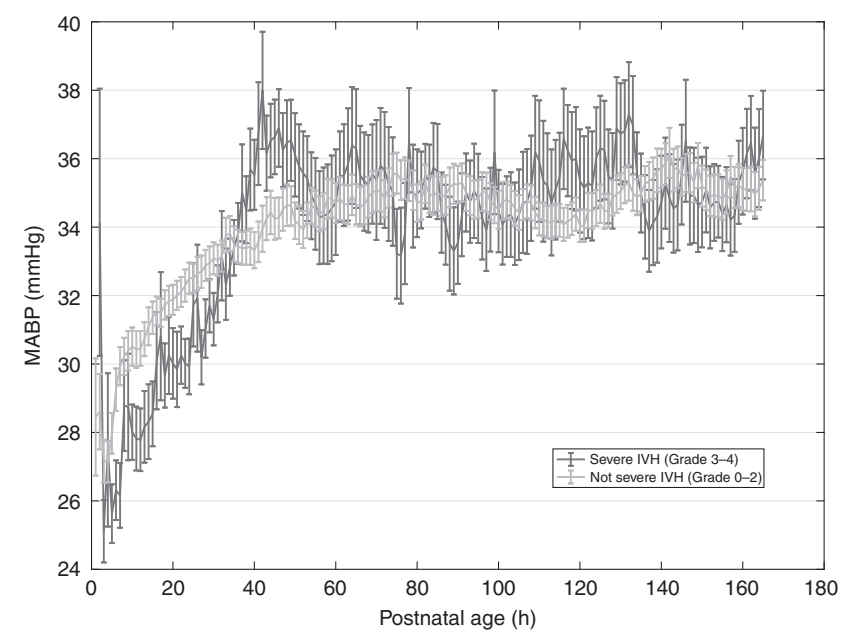

Fig. 1 Hourly plot of mean \pm SEM of MABP for infants with severe IVH and without severe IVH. Note the significantly greater fluctuations of the severe IVH group

\section{MABP distribution}

The mean MABP over the 7-day period was not different between infants with severe IVH, without severe IVH, and no detectable IVH (34.1 vs. 33.8 vs. $34.2 \mathrm{~mm} \mathrm{Hg}, p=0.58$ ), but visual comparison of the hourly mean MABP shows markedly different patterns. Infants without severe IVH had a gradual increase in MABP while those with severe IVH had a markedly lower MABP for the first $40 \mathrm{~h}$ followed by significant high-amplitude fluctuations over the next 48-72 $\mathrm{h}$ (Fig. 1). When using the Zubrow definition of hypotension (MABP $<$ GA in weeks), infants with severe IVH spent a greater proportion of time with hypotension than those without severe IVH and no detectable IVH ( $29 \%$ vs. $23 \%$ vs. $12 \%)$, although this difference was not statistically significant $(p=0.78)$. When using the Miall-Allen definition of hypotension (MABP $<30 \mathrm{~mm} \mathrm{Hg}$, regardless of GA), infants with severe IVH spent slightly less time with hypotension than infants without severe IVH and no IVH ( $21 \%$ vs. $30 \%$ vs. $28 \%$ ), although this difference again was not statistically significant $(p=0.54)$.

Threshold identification and model development A visual inspection of grouped box and whisker plots (Fig. 2) reveals obvious qualitative differences; the infants with severe IVH spend a proportionally greater amount of time at the extreme ends of the distribution, particularly $<26$ and $>39 \mathrm{~mm} \mathrm{Hg}$. This finding is in contrast to the infants without IVH or with mild IVH who spend a proportionally greater amount of time in the middle range of BPs.

The mean percentage of the recording at or beyond each bin was systematically evaluated in order to identify candidate thresholds for the final model, defined by the MABP bin with the greatest AUC in univariate analysis. When comparing infants with severe IVH to those without severe IVH, these MABP thresholds are noted at $\leq 23 \mathrm{~mm} \mathrm{Hg}$ (AUC $=0.612$ ) and $\geq 46 \mathrm{~mm}$ $\mathrm{Hg}$ (AUC $=0.624$ ) (Table 2a). When comparing infants with severe IVH to those without detectable IVH, the maximal AUC occurs at the same BP thresholds, $\leq 23 \mathrm{~mm} \mathrm{Hg}$ (AUC $=0.664$ ) and $\geq 46 \mathrm{~mm}$ $\mathrm{Hg}(\mathrm{AUC}=0.637$ ) (Table 2b). When the burden of MABP values above and below these thresholds are combined, the discriminatory power of the models become even stronger (AUC $=0.650$ and 0.698 , respectively).

On average, infants without IVH spent $2 \%$ of the recording $(202 \mathrm{~min})$ with $\mathrm{MABP} \leq 23 \mathrm{~mm} \mathrm{Hg}$ while infants with severe IVH spent twice as much time (4\% of the recording, $404 \mathrm{~min}$ ) below the same threshold. Similarly, the infants without IVH spent an average of $3 \%$ of the recording ( $303 \mathrm{~min}$ ) with MABP $\geq 46 \mathrm{~mm} \mathrm{Hg}$

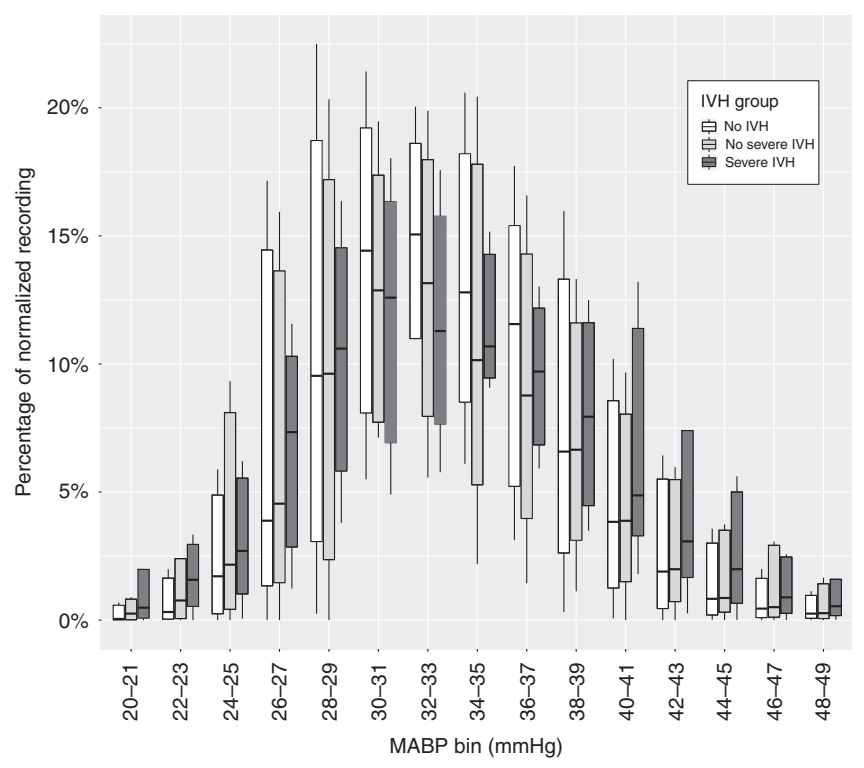

Fig. 2 Grouped box plots demonstrating the burden of MABP at each bin by group. Of note, infants with severe IVH spent a significantly greater proportion of time with MABP at the extremes of the distribution, both high and low

Table 2. High and low MABP burdens: (a) no severe IVH vs. severe IVH and (b) no IVH vs. severe IVH

\begin{tabular}{|c|c|c|c|}
\hline \multirow{2}{*}{$\begin{array}{l}\text { Threshold }(\mathrm{mm} \mathrm{Hg}) \\
\text { (a) No severe IVH vs. severe IVH }\end{array}$} & \multicolumn{2}{|c|}{$\begin{array}{l}\text { Mean normalized } \\
\text { percentage of recording } \\
\text { beyond threshold }\end{array}$} & \multirow[t]{2}{*}{ AUC } \\
\hline & No severe IVH & Severe IVH & \\
\hline$\leq 21$ & 0.01 & 0.02 & 0.634 \\
\hline$\leq 23$ & 0.03 & 0.04 & 0.637 \\
\hline$\leq 25$ & 0.07 & 0.09 & 0.612 \\
\hline$\leq 27$ & 0.14 & 0.17 & 0.582 \\
\hline$\geq 40$ & 0.16 & 0.20 & 0.606 \\
\hline$\geq 42$ & 0.10 & 0.13 & 0.616 \\
\hline$\geq 44$ & 0.06 & 0.08 & 0.614 \\
\hline$\geq 46$ & 0.03 & 0.04 & 0.624 \\
\hline$\geq 48$ & 0.01 & 0.02 & 0.619 \\
\hline$\leq 23$ and $\geq 46$ & 0.08 & 0.12 & 0.659 \\
\hline (b) No IVH vs. severe IVH & No IVH & Severe IVH & \\
\hline$\leq 21$ & 0.01 & 0.02 & 0.650 \\
\hline$\leq 23$ & 0.02 & 0.04 & 0.664 \\
\hline$\leq 25$ & 0.06 & 0.09 & 0.638 \\
\hline$\leq 27$ & 0.14 & 0.16 & 0.606 \\
\hline$\geq 40$ & 0.15 & 0.20 & 0.615 \\
\hline$\geq 42$ & 0.09 & 0.13 & 0.629 \\
\hline$\geq 44$ & 0.05 & 0.08 & 0.628 \\
\hline$\geq 46$ & 0.03 & 0.04 & 0.637 \\
\hline$\geq 48$ & 0.01 & 0.02 & 0.631 \\
\hline$\leq 23$ and $\geq 46$ & 0.06 & 0.12 & 0.698 \\
\hline
\end{tabular}

while infants with severe injury spent $4 \%$ of the recording (404 min) above the same level.

In the linear regression model, inotrope exposure (odds ratio (OR) $1.73, p=0.02$ ), antenatal steroid exposure (OR $0.47, p<0.01$ ), and the burden of MABP extremes (OR 19.15, $p=0.01$ ) were independently predictive of IVH risk and severity, when controlling 
Table 3. Regression model output

\begin{tabular}{lcc}
\hline Variable & $\begin{array}{l}\text { Adjusted OR } \\
(95 \% \mathrm{Cl})\end{array}$ & $p$ Value \\
\hline Gestational age at birth & $0.92(0.79-1.10)$ & 0.29 \\
Birth weight & $1.00(0.99-1.01)$ & 0.15 \\
Antenatal steroids & $0.47(0.27-0.82)$ & $<0.01^{*}$ \\
Inotrope exposure & $1.73(1.08-2.78)$ & $0.02^{*}$ \\
Percentage of time spent with MABP & $19.15(1.75-88.96)$ & $0.01^{*}$ \\
beyond thresholds & & \\
Model: IVH grade $\sim \mathrm{GA}+\mathrm{BW}+$ antenatal steroids + inotrope exposure \\
+ \% outside threshold \\
Model multiple $R$-squared $=0.164$, Model $F=5.42, p<0.01$ & \\
\hline
\end{tabular}

*Denotes significance at $p<0.05$

Table 4. Interaction model investigation

\begin{tabular}{lcc}
\hline Variable & $T$ statistic & $p$ Value \\
\hline Gestational age at birth & -0.89 & 0.37 \\
Birth weight & 1.60 & 0.11 \\
Antenatal steroids & -2.84 & $<0.01^{*}$ \\
Inotrope exposure & 1.71 & 0.09 \\
Percentage of time spent with MABP $\leq 23 \mathrm{~mm} \mathrm{Hg}$ & 1.74 & 0.09 \\
Percentage of time spent with MABP $\geq 46 \mathrm{~mm} \mathrm{Hg}$ & 0.76 & 0.44 \\
MABP interaction & 1.98 & $0.04^{*}$ \\
\hline *Denotes significance at $p<0.05$ & & \\
\hline
\end{tabular}

for GA at birth and BW. See Table 3 for complete regression model results.

As the distribution of BPs in Fig. 1 demonstrates, there is a distinct MABP pattern for infants who are later found to have severe IVH-lower BP in the first $36 \mathrm{~h}$ (the highest risk period of IVH) followed by higher, more variable BPs thereafter. When the "low" and "high" burdens are added separately to the model, neither main effect is statistically significant, but their interaction is strongly significant $(p<0.01)$ suggesting that this particular pattern is uniquely associated with IVH (Table 4).

\section{DISCUSSION}

Defining the "normal" BP of a preterm infant remains one of the most challenging conundrums in neonatology. Although the BP itself is a dynamic factor, it is only one piece of a complex system of cardiac output, vascular autoregulation, and metabolic demand. $^{20}$ Previous normative studies have helped to outline what the BP of infants with good outcomes is, but not necessarily what it needs to be. IVH represents the most consequential outcome of a failure to keep the BP within these boundaries. The fragile germinal matrix of the preterm brain is poorly suited to handle such fluctuation. Furthermore, clinical interventions such as the administration of intravenous fluids, inotropic agents, and postnatal corticosteroids introduce additional instability to the BP, creating the potential for therapeutic overshoot and possibly contributing to IVH risk.

We have hypothesized that IVH results from an excessive burden of BP values outside of a narrow envelope. The MABP is an ideal metric for this investigation, as it is a single measure reflective of the composite influence of cardiac output, volume status, and $\mathrm{pH}$, among others. When examined over time, the value only increases; infants with severe IVH have lower MABP values as well as substantially more unstable values, as clearly demonstrated in Fig. 1. When examined as a cumulative burden in this data-rich cohort, a clear pattern emerges; not only do infants with severe IVH spend more time at the extremes of MABP, but clear transition points can be identified where the severe IVH group makes up the majority of observations beyond those points. Furthermore, there is a very specific pattern of early low BP followed by widely variable high BP. Given the statistically significant interaction, both must occur for severe IVH to result.

These results offer a hint toward possible "guard rails" for BP management, namely, that maintenance of MABP within the $23-46 \mathrm{~mm} \mathrm{Hg}$ window may be the ideal target range in preterm infants $<30$ weeks gestation. It is crucial to emphasize that infants with good outcomes do spend time outside of this narrow window, nearly $8 \%$ of the recording (approximately $14 \mathrm{~h}$ in the first week) on average. In a secondary analysis of this cohort, infants without exposure to inotropes spent an average of $4 \%$ of the recording (nearly $7 \mathrm{~h}$ ) with an $M A B P \geq 46 \mathrm{~h} \mathrm{~mm} \mathrm{Hg}$, suggesting that MABP values this high routinely occur as a part of the natural progression of the BP rather than from over-treatment. However, persistent and prolonged hypotension or hypertension should be a warning sign prompting further investigation and possibly intervention.

We have also demonstrated that the burden of hypotension by traditional BP definitions (MABP $<G A$ in weeks, MABP $<30 \mathrm{~mm}$ $\mathrm{Hg}$ ) is not associated with severe IVH. This is not surprising. The Zubrow study ${ }^{9}$ utilized oscillometric measurements of BP three times daily. In addition to significant issues with test-retest reliability for non-invasive $\mathrm{BP}$ measurement, the sampling rate is wholly insufficient to capture the widely varying BP fluctuations characteristic of the first week of a preterm infant's life. The MiallAllen study ${ }^{10}$ does not suffer from the same issues as invasive arterial measurements were used. The authors noted that $60 \mathrm{~min}$ of exposure to an MABP $<30 \mathrm{~mm} \mathrm{Hg}$ was associated with an increased risk of severe hemorrhage. The present study demonstrates otherwise; infants without severe hemorrhage spent slightly more time with MABP $<30 \mathrm{~mm} \mathrm{Hg}$ (30\% of recording) compared to those with severe hemorrhage (21\%). One limitation of the Miall-Allen study is that it was performed $>30$ years ago, in a very different era of neonatology before the widespread use of antenatal steroids and surfactant replacement therapy. It is possible that changes in perinatal management permit wider latitude in acceptable BPs than was previously possible. It is also possible that the 30 infants in that study were not fully representative of the premature population as a whole.

There are important limitations to consider in the present study. One potential concern is selection bias introduced by the inclusion criteria, namely, that infants in modern neonatal care who have arterial lines in place for at least 7 days are likely to be more immature and more sick than infants with fewer line days (or no arterial line at all), indeed only about $10 \%$ of the total infants admitted to the two NICUs met this stringent criteria. Although this was an intentional study design decision, to include the entire risk period for $\mathrm{IVH}$, future studies should confirm that similar upper and lower boundaries are found for infants with shorter duration recordings. Second, these findings are based on a single metric (MABP), by design. It is possible, and indeed likely, that the inclusion of other physiologic measures will add additional value but will be more difficult to obtain and translate into clinical practice. Third, the timing of cranial ultrasounds was entirely at the discretion of the clinical team and was highly variable. As a result, discrete timing of the hemorrhage and clear identification of preand post-hemorrhage periods was not possible. Future studies should focus on consistent and frequent imaging (ideally once per day) to more clearly delineate these periods. Finally, a note of caution should be urged against over-interpretation of the presented data. These results represent the findings of infants at two institutions over a short interval of time. These results should 
be evaluated at other centers with different demographic characteristics and clinical practices to ensure their robustness and before these thresholds are implemented in clinical practice.

Given the infrequent nature of cranial ultrasounds, this study design does not permit investigation into the burdens of hypotension and hypertension before and after IVH. The hypothesis of this study, supported by previous reports, ${ }^{1}$ is that hypotension is a causative factor for IVH. However, it is possible that the hemorrhage could itself cause hemodynamic instability, manifested as hypotension. Additionally, the elevated BP values may be deleterious or may reflect the need for higher cerebral blood flow in the post-hemorrhage state, driven by elevated intracranial pressure, as is seen in adult stroke. ${ }^{21,22}$ Future investigation with more frequent ultrasounds and more precisely timed hemorrhages will be better suited to answer these questions.

\section{Conclusions}

In this study, we have demonstrated that infants who develop severe IVH have substantially more unstable BPs in the first week of life. Evaluating the distribution of BP measurements, candidate thresholds of optimal BP were derived. Infants with severe IVH have a consistent pattern of low BP initially followed by an "overshoot" and instability. It remains unclear as to where in this pattern severe IVH occurs. Follow-up studies with close attention to the discrete timing of IVH may offer the possibility of a therapeutic BP target.

\section{ACKNOWLEDGEMENTS}

This work was supported by the following grants: Washington University Institute of Clinical and Translational Sciences KL2 Training Program (NIH/NCATS KL2 TR000450); The Barnes-Jewish Hospital Foundation and the Washington University Institute of Clinical and Translational Sciences Clinical and Translational Funding Program $(\mathrm{NIH} /$ NCATS UL1 TR000448); and the National Institutes of Health grant numbers K23 NS111086, R01 HD072071, and R01 HL133708.

\section{AUTHOR CONTRIBUTIONS}

Z.A.V. provided the initial design of the work, collected data, performed data analysis, and wrote the first draft of the article. A.A.F., K.D.F., and A.M.M. provided input on the design of the work, assisted in data analysis, and provided critical revision of the article. S.Z., A.R., M.A., and H.V.W. assisted in data collection and analysis and provided critical revision of the article. All the authors have reviewed and agree with the final version to be published.

\section{ADDITIONAL INFORMATION}

Competing interests: The authors declare no competing interests.

Publisher's note Springer Nature remains neutral with regard to jurisdictional claims in published maps and institutional affiliations.

\section{REFERENCES}

1. Volpe, J. J. Neurology of the Newborn 5th edn (Saunders/Elsevier, Philadelphia, 2008).

2. Ment, L. R. et al. Antenatal steroids, delivery mode, and intraventricular hemorrhage in preterm infants. Am. J. Obstet. Gynecol. 172, 795-800 (1995).

3. Stoll, B. J. et al. Neonatal outcomes of extremely preterm infants from the NICHD Neonatal Research Network. Pediatrics 126, 443-456 (2010).

4. Vesoulis, Z. A., Liao, S. M., Trivedi, S. B., Ters, N. E. \& Mathur, A. M. A novel method for assessing cerebral autoregulation in preterm infants using transfer function analysis. Pediatr. Res. 79, 453-459 (2016).

5. Soul, J. S. et al. Fluctuating pressure-passivity is common in the cerebral circulation of sick premature infants. Pediatr. Res. 61, 467-473 (2007).

6. Chock, V. Y., Ramamoorthy, C. \& Van Meurs, K. P. Cerebral autoregulation in neonates with a hemodynamically significant patent ductus arteriosus. J. Pediatr. 160, 936-942 (2012)

7. Alderliesten, T. et al. Cerebral oxygenation, extraction, and autoregulation in very preterm infants who develop peri-intraventricular hemorrhage. J. Pediatr. 162 698.e2-704.e2 (2013).

8. Tsuji, M. et al. Cerebral intravascular oxygenation correlates with mean arterial pressure in critically ill premature infants. Pediatrics 106, 625-632 (2000).

9. Zubrow, A. B., Hulman, S., Kushner, H. \& Falkner, B. Determinants of blood pressure in infants admitted to neonatal intensive care units: a prospective multicenter study. Philadelphia Neonatal Blood Pressure Study Group. J. Perinatol. 15, 470-479 (1995)

10. Miall-Allen, V. M., de Vries, L. S. \& Whitelaw, A. G. Mean arterial blood pressure and neonatal cerebral lesions. Arch. Dis. Child. 62, 1068-1069 (1987).

11. Bada, H. S. et al. Mean arterial blood pressure changes in premature infants and those at risk for intraventricular hemorrhage. J. Pediatr. 117, 607-614 (1990).

12. Vesoulis, Z. A., El Ters, N. M., Wallendorf, M. \& Mathur, A. M. Empirical estimation of the normative blood pressure in infants $<28$ weeks gestation using a massive data approach. J. Perinatol. 36, 291-295 (2016).

13. Lee, J., Rajadurai, V. S. \& Tan, K. W. Blood pressure standards for very low birthweight infants during the first day of life. Arch. Dis. Child. Fetal Neonatal Ed. 81, F168-F170 (1999).

14. Hegyi, T. et al. Blood pressure ranges in premature infants. I. The first hours of life. J. Pediatr. 124, 627-633 (1994).

15. St. Peter, D., Gandy, C. \& Hoffman, S. B. Hypotension and adverse outcomes in prematurity: comparing definitions. Neonatology 111, 228-233 (2017).

16. Alderliesten, T. et al. Hypotension in preterm neonates: low blood pressure alone does not affect neurodevelopmental outcome. J. Pediatr. 164, 986-991 (2014).

17. Lou, H. C., Skov, H. \& Pedersen, H. Low cerebral blood flow: a risk factor in the neonate. J. Pediatr. 95, 606-609 (1979).

18. Ment, L. R., Ehrenkranz, R. A., Lange, R. C., Rothstein, P. T. \& Duncan, C. C. Alterations in cerebral blood flow in preterm infants with intraventricular hemorrhage. Pediatrics 68, 763-769 (1981).

19. Van Bel, F., Van de Bor, M., Stijnen, T., Baan, J. \& Ruys, J. H. Aetiological rôle of cerebral blood-flow alterations in development and extension of periintraventricular haemorrhage. Dev. Med. Child Neurol. 29, 601-614 (1987).

20. Noori, S., McCoy, M., Anderson, M. P., Ramji, F. \& Seri, I. Changes in cardiac function and cerebral blood flow in relation to peri/intraventricular hemorrhage in extremely preterm infants. J. Pediatr. 164, 264.e3-270.e3 (2014).

21. Wallace, J. D. Blood pressure after stroke. JAMA 246, 2177-2180 (1981).

22. Leonardi-Bee, J., Bath, P. M. W., Phillips, S. J. \& Sandercock, P. A. G. Blood pressure and clinical outcomes in the International Stroke Trial. Stroke 33, 1315-1320 (2002). 